\title{
A radio continuum and neutral hydrogen counterpart to the IRAS Vela shell
}

\author{
J. C. Testori ${ }^{1, \star}$, E. M. Arnal ${ }^{1,2, \star}$, R. Morras ${ }^{1,2, \star}$, E. Bajaja ${ }^{1, \star}$, W. G. L. Pöppel ${ }^{1, \star}$, and P. Reich ${ }^{3}$ \\ 1 Instituto Argentino de Radioastronomía (IAR), C.C. No. 5, 1894 Villa Elisa, Argentina \\ e-mail: jtestori@iar.unlp.edu.ar \\ 2 Facultad de Ciencias Astronómicas y Geofísica Universidad Nacional de La Plata, Paseo del Bosque s/n, 1900 La Plata, Argentina \\ 3 Max-Planck-Institut für Radiastronomie, Auf dem Hügel 69, 53121 Bonn, Germany
}

Received 11 January 2006 / Accepted 2 June 2006

\section{ABSTRACT}

\begin{abstract}
Aims. We look for radio-continuum and atomic neutral hydrogen (HI) counterparts of the IRAS Vela shell (IVS).
Methods. Neutral hydrogen (HI) and $1410 \mathrm{MHz}$ radio continuum surveys carried out with the Instituto Argentino de Radioastronomía (IAR) dishes are the main databases used in this investigation.

Results. A good morphological correlation was found between arc-shaped structures at radio wavelengths and in the $100 \mu \mathrm{m}$ IR emission. This correlation is observed at velocities of the HI between -17.5 and $-2.1 \mathrm{~km} \mathrm{~s}^{-1}$, in agreement with the velocities of the molecular gas detected in the region. The radial distribution of the different components is consistent with the presence of an expanding shell that is being ionized from inside. Assuming a distance of $400 \mathrm{pc}$, the amount of ionized and atomic gas associated with the IVS is $2.3 \times 10^{4}$ and $6.8 \times 10^{4}$ solar masses, respectively. The origin of the expansion of the IVS could not be identified.
\end{abstract}

Key words. ISM: clouds - Galaxy: kinematics and dynamics - radio lines: ISM

\section{Introduction}

The IRAS Vela shell (IVS) is a prominent far-IR ring-like feature first noticed on the 25, 60, and $100 \mu \mathrm{m}$ IRAS maps by Sahu (1992). According to this author this feature is centred at about $(l, b)=\left(263^{\circ},-7^{\circ}\right)$, has an angular radius of $\sim 7.5$, and may be surrounding the OB-association Vel OB2 (Sahu 1992). Unfortunately, the IVS is not an isolated feature in the sky, but appears projected onto the southwestern boundary of the Gum nebula that, with its $36^{\circ}$ in diameter, has one of the largest angular extents known for an $\mathrm{H}_{\alpha}$ emission object.

In Fig. 1, the IVS is easily recognisable as a shell-like feature. In the same figure an IR emission-intensity asymmetry is also noticeable (Sahu 1992). Indeed, the IR emission from the region $265^{\circ} \leq l \leq 275^{\circ}$ and $-15^{\circ} \leq b \leq 0^{\circ}$ is more intense than the one observed $255^{\circ} \leq l \leq 265^{\circ}$ and $-15^{\circ} \leq b \leq 0^{\circ}$. Through the gas-to-dust relationship, this may indicate that the second region may be comparatively deficient in dust and gas compared to the former. Close to the galactic plane, the IVS is not clearly traceable due to confusion with far-IR emission arising from the galactic plane itself. Based on low angular-resolution observations, extended and strong $\mathrm{CO}$ emission was found to be confined to the galactic region $260^{\circ} \leq l \leq 270^{\circ}$ and $|b| \leq 2^{\circ}$ (Murphy \& May 1991). This intense CO feature is usually referred to as the Vela molecular ring (VMR) and it is composed of at least four different molecular concentrations, which are best observed within the velocity ${ }^{1}$ range of 5.9 to $9.8 \mathrm{~km} \mathrm{~s}^{-1}$. The dust associated with this gaseous component is responsible for the strong IR emission seen in the VMR zone. The distances of the

* Member of the Carrera del Investigador Científico of the Consejo National de Investigaciones Científicas y Técnicas of Argentina.

${ }^{1}$ All velocities refer to the local standard of rest (LSR).

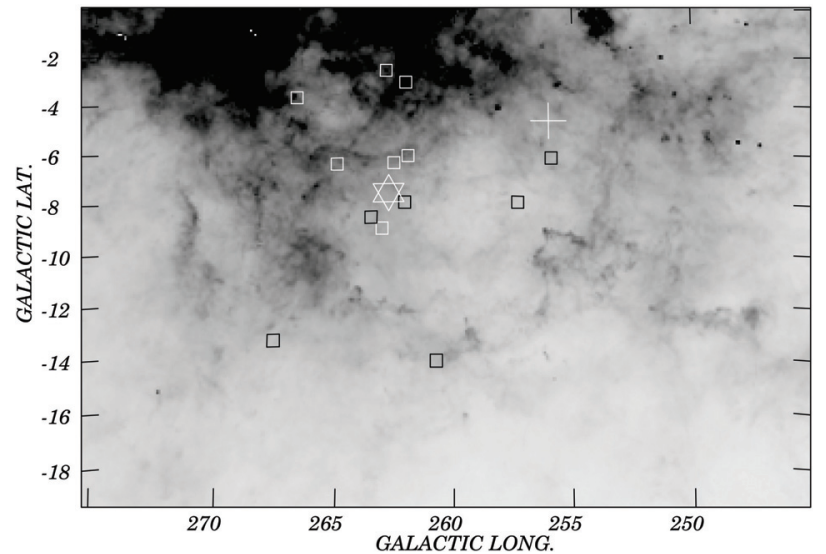

Fig. 1. Sky distribution of the far-IR $100 \mu \mathrm{m}$ emission in the IRAS Vela shell region, as provided by the SkyView Facility. The star symbol marks the location of $\gamma^{2}$ Vel, while the position of $\zeta$ Puppis is given by a cross. Black and white square boxes represent Vel OB2 members, as in de Zeeuw et al. (1999), whose spectral types are earlier than B3 V.

different concentrations vary between $\sim 1 \mathrm{kpc}$ and $\sim 2.6 \mathrm{kpc}$ (Murphy \& May 1991).

The IVS can also be traced by the spatial distribution of dark cloud and cometary globules (CG) and by the angular distribution of the young stellar objects (YSOs). The former is apparent in the maps of Feitzinger \& Stüwe (1984) in their study of the Milky Way dark-cloud distribution. In particular, the catalogued CG are seen on ESO plates as "cometary-like" dark clouds having a dense head that is completely opaque to the background starlight and faint luminous tails. The heads often have bright rims on the side pointing to the centre of the IVS, while the tails are always pointing away from the central region. These CG and 
dark clouds have been known for more than 20 years, and since then it has been recognised that most of them are somehow related to each other and form a complex ring-like structure that closely follows the IVS (Zealey 1979; Reipurth 1983).

Prusti (1992) using the IRAS Point Source Catalogue (IPSC) and certain colour and "crowding" criteria, selected $\sim 1800$ candidates for low-mass YSOs in the region. Remarkably enough, the IVS $100 \mu \mathrm{m}$ structure is closely followed by their spatial distribution. The kinematics of the IVS is a controversial issue. Based on the kinematics, derived from CO $J=1 \rightarrow 0$ observations towards $\sim 100$ IR-sources that are projected onto the IVS, Rajagopal \& Srinivasan (1998) conclude that the IVS is expanding at a velocity of $\sim 13 \mathrm{~km} \mathrm{~s}^{-1}$ from a common centre located at $(l, b)=\left(260^{\circ},-4^{\circ}\right)$.

A similar conclusion was reached by Sahu \& Sahu (1993) based on $\lambda \lambda 6548,6584$ [NII] high-resolution spectroscopy carried out at 18 positions scattered across the "face" of the Gum nebula. They claimed that only positions within the IVS show double-peaked components in [NII], interpreting this line splitting as arising from ionized gas expanding at $10 \pm 2 \mathrm{~km} \mathrm{~s}^{-1}$. Since most of the observations carried out towards the Gum nebula failed to report expanding motions (Hippelein \& Weinberger 1975; Wallerstein et al. 1980), Sahu \& Sahu (1993) interpreted the different kinematical behaviour between the Gum nebula and the IVS as strong evidence in favour of their both being physically unrelated to each other. It ought to be mentioned that this interpretation is challenged by previous $\mathrm{H}_{\alpha}$ and $\mathrm{N}[\mathrm{II}]$ observations carried out by Reynolds (1976a), since his data revealed double components at positions located outside the IVS boundary.

On the other hand, Woermann et al. (2001) studied the kinematics of the Gum nebula using OH-1667 MHz line emission data. Though they find an expanding shell of molecular gas covering all of the $\mathrm{H}_{\alpha}$ emission of the Gum, they find no evidence in their data to claim that the IVS is a physical structure unrelated to the Gum nebula.

Thus, until this point is properly settled, one should take the argument of Sahu \& Sahu (1993), in favour of both an IVS expansion and a different spatial location of the Gum nebula and the IVS, with some caution. In the past, a gaseous counterpart to the IVS was looked for, but studies of Sahu (1992) and Reynoso \& Dubner (1997) using different HI databases failed to find such a feature.

In this paper we present the results of a new search aimed at finding a gaseous counterpart, either atomic or ionized, to the IVS. This study was carried out using a new high-sensitivity $1420 \mathrm{MHz}$ continuum survey (Testori et al. 2001; Reich et al. 2001) and a new high-sensitivity HI survey (Arnal et al. 2000; Bajaja et al. 2005). The main characteristics of the databases are briefly outlined in Sect. 2, the results are given in Sect. 3, and the discussion in Sect. 4. Conclusions are presented in Sect. 5.

\section{Databases}

We relied mainly on four databases:

i) a high sensitivity Hi survey of the southern sky. Arnal et al. (2000) describe the motivation and observing strategy of the IAR HI survey, Bajaja et al. (2005) describe the strayradiation correction applied to the data, and Kalberla et al. (2005) present the data, as combined the northern material of the Leiden/Dwingeloo Survey (LDS), via a link to the CDS; ii) a total power high-sensitivity $1420 \mathrm{MHz}$ southern hemisphere continuum survey (Testori et al. 2001; Reich et al. 2001);

iii) the IRAS data at $100 \mu \mathrm{m}$;

iv) the Southern H-Alpha Sky Survey Atlas ${ }^{2}$ (SHASSA) (Gaustad et al. 2001).

The $1420 \mathrm{MHz}$ continuum survey was carried out using one of the two $30 \mathrm{~m}$-dishes available at the Instituto Argentino de Radioastronomía. This survey is absolutely calibrated, has an angular resolution of 35.4 , and a sensitivity of $\sim 50 \mathrm{mK}(3 \sigma)$ in fullbeam brightness temperature. The HI survey was carried out using a $30 \mathrm{~m}$ dish $\left(H P B W \sim 30^{\prime}\right)$ on a $0.5 / \cos b \times 0.5$ grid in galactic coordinates. The survey covers the velocity interval -400 to $450 \mathrm{~km} \mathrm{~s}^{-1}$, and the velocity resolution is $\sim 1.3 \mathrm{~km} \mathrm{~s}^{-1}$. The final rms noise of a single profile is $\sim 70 \mathrm{mK}$ on the brightness temperature scale. This scale is accurate to $5 \%$. The strayradiation corrected version of the HI survey (see Bajaja et al. 2005, for details) has been used in this paper. Finally, the IRAS $100 \mu \mathrm{m}$ map was retrieved using SkyView ${ }^{3}$. The $100 \mu \mathrm{m}$ IRAS data have an angular resolution of around $5^{\prime}$.

\section{Observational results}

\subsection{Continuum emission at $1420 \mathrm{MHz}$}

The large angular-scale continuum emission present in the area of the IVS was filtered out by using the "BackGround Filtering" method (BGF) described by Sofue \& Reich (1979). A $4^{\circ} \times 4^{\circ}$ filtering beam was applied, and an overlay of the resulting $1420 \mathrm{MHz}$ continuum emission and the $100 \mu \mathrm{m}$ IRAS emission is shown in Fig. 2. The overall radio-continuum emission depicts a remarkable morphological correspondence with the IRAS $100 \mu \mathrm{m}$ emission delineating the IVS. In particular, the shell-like $100 \mu \mathrm{m}$ emission marking the southern border of the IVS has a clear counterpart in the radio continuum. To our knowledge this is the first time that such spatial correlation is noticed.

Using both the $408 \mathrm{MHz}$ (Haslam et al. 1982), and the $1420 \mathrm{MHz}$ (Reich et al. 2001) continuum surveys, the brightness-temperature spectral index $\beta$ of the IVS was determined (Testori 2001). A mean weighted value of $\beta=2.17 \pm 0.07$ $\beta\left(T_{b} \propto v^{-\beta}\right)$ was obtained. This indicates that the continuum emission is mostly thermal. A weak nonthermal component was reported by Duncan et al. (1995).

Due to the contaminating effect of Vela SNR, the flux density of the continuum feature was measured in only $\sim 65 \%$ of its angular extent. Assuming that there is continuum emission associated with the IVS at the same level over all the remaining area, we derived a total flux density of $S_{v}=500 \pm 70 \mathrm{Jy}$. We used the model of Mezger \& Henderson (1967, MH from here) to infer the physical parameters of the ionized gas. The spatial geometry of the continuum feature was approximated by a spatially spherical shell of inner and outer Gaussian half widths $\theta_{\mathrm{i}}$ and $\theta_{\mathrm{o}}$, respectively. Hence the term $\theta^{1.5}$, the measured half-power Gaussian width in the Eqs. (6) and (7) of MH, should be replaced by $\left(\theta_{\mathrm{o}}^{3}-\theta_{\mathrm{i}}^{3}\right)^{0.5}$. From our data we obtained a value of 840 and 660 arcmin for $\theta_{\mathrm{o}}$ and $\theta_{\mathrm{i}}$, respectively. The electron

\footnotetext{
2 The Southern H-Alpha Sky Survey is supported by the National Science Foundation.

3 The SkyView facility is operated at High Energy Astrophysics Sciences Archive Research Center (HEASARC) at the GSFC Laboratory for High Energy Astrophysics.
} 


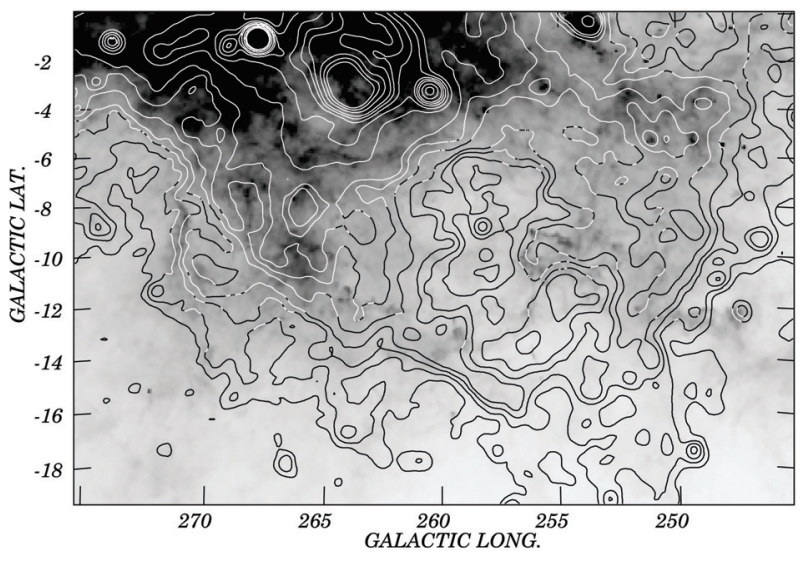

Fig. 2. Continuum map (contour lines) superimposed on a $100 \mu \mathrm{m}$ IRAS image (gray shadings). The lowest contour line and the contour spacing are between $50 \mathrm{mK}$ and $200 \mathrm{mK}$. The temperature is ful-beam brightness temperature. Above the contour line of $200 \mathrm{mK}$, the contour step is between $100 \mathrm{mK}$ and $500 \mathrm{mK}$, then the contour step is $500 \mathrm{mK}$ till $3000 \mathrm{mK}$ and from there onwards to $1000 \mathrm{mK}$. The gray scale spans the range from 0 (white) to 80 (black) $\mathrm{MJy} / \mathrm{sr}$.

density $\left(n_{\mathrm{e}}\right)$, the mass of ionized gas $\left(M_{\mathrm{i}}\right)$, and the total number of UV ionizing photons $\left(N_{\mathrm{u}}\right)$ needed to keep the source ionized (Chaisson 1976) can be derived from the measured total flux density $S_{v}$ and the adopted source geometry. For simplicity, we adopted $T_{\mathrm{e}}=10^{4} \mathrm{~K}$ for these calculations because the parameters of an HII region only weakly depend on the assumed electron temperature.

Following $\mathrm{MH}$, an electron density $n_{\mathrm{e}} \simeq 0.73 d^{-0.5} \mathrm{~cm}^{-3}$ and a mass of ionized gas $M_{\mathrm{i}}=2.26 \times 10^{5} d^{2.5} M_{\odot}$ were determined, with $d$ the distance to IVS in kpc. The number of Lyman continuum photons needed to keep the source ionized is $N_{\mathrm{u}} \sim 3.9 \times$ $10^{49} d^{2} \mathrm{~s}^{-1}$. The emission measure (EM) of the shell was calculated following the procedure of Cichowolski et al. (2001). Those regions of the shell having the weakest excess brightness temperature, about $15 \mathrm{mK}$, have an excess EM of $\sim 100 \mathrm{pc} \mathrm{cm}^{-6}$, while the brighter regions, whose excess brightness temperature is about 400 to $500 \mathrm{mK}$, have an excess emission measure of 250 to $320 \mathrm{pc} \mathrm{cm}^{-6}$. These values are consistent with those reported by Reynolds (1976b, $150 \leq E M \leq 500 \mathrm{pc} \mathrm{cm}^{-6}$ ), Woermann et al. (2000) $\left(220 \leq E M \leq 470 \mathrm{pc} \mathrm{cm}^{-6}\right)$, and Testori $\left(2001,100 \leq E M \leq 400 \mathrm{pc} \mathrm{cm}^{-6}\right)$.

The spatial correlation between the IVS radio continuum emission at $1420 \mathrm{MHz}$ and the $\mathrm{H}_{\alpha}$ emission (as provided by the SHASSA database) is shown in Fig. 3. The correlation between both emissions is quite remarkable, implying that very likely the same gas is given rise to both emissions.

\subsection{Neutral hydrogen emission}

Before undertaking the task of searching for a neutral hydrogen counterpart of the IVS, we briefly summarize the main results of previous HI studies performed in the region under study. The goal of this summary is 1) to show the reader the variety of HI structures that are already known to exist in the area under study; and 2) to help to define those regions of the sky where the HI emission arising from the neutral hydrogen gas, which could be associated with the IVS, will be less confused by the presence of HI structures unlikely to be related to the IVS.

Using the Weaver \& Williams (1973) HI survey, Heiles (1979) studied the region $20^{\circ} \leq l \leq 250^{\circ}$ and $b \leq\left|10^{\circ}\right|$ and found several arcs of $\mathrm{HI}$ emission that change size with velocity in the

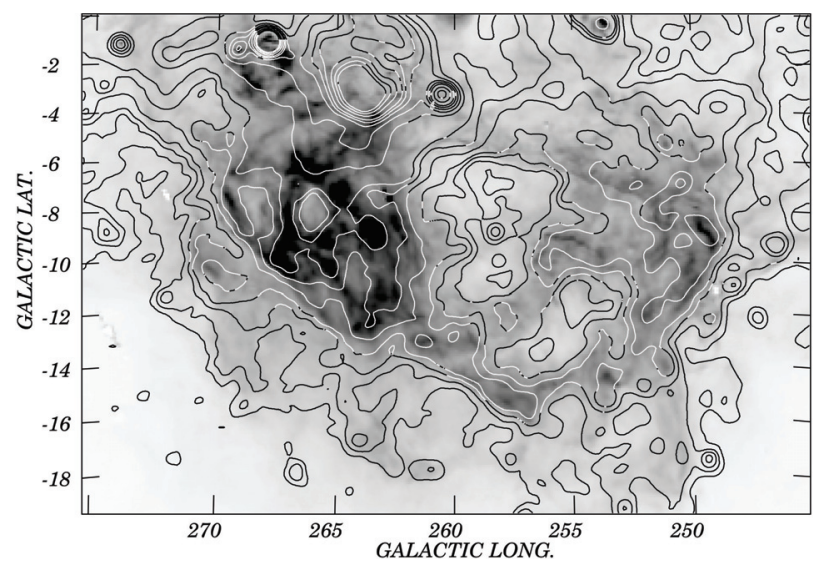

Fig. 3. Overlay of the continuum emission (contour lines) and the SHASSA $\mathrm{H}_{\alpha}$ emission (gray scale). The contours are as in Fig. 2. The gray scale covers the range from $0 \mathrm{R}$ (light gray) to $3000 \mathrm{R}$ (dark gray).

manner expected for an expanding shell. Three giant shells were discovered in the region $240^{\circ} \leq l \leq 250^{\circ}$ and $-20^{\circ} \leq b \leq+1^{\circ}$. Two of them, GS241-04-05 and GS242-01+11, are stationary (their angular size does not change with velocity), while the third one, GS242-03+37, is expanding. The shell GS241-04-05 has a size of $16^{\circ} \times 19^{\circ}(\Delta l \times \Delta b)$ and spans the velocity range -7 to $-3 \mathrm{~km} \mathrm{~s}^{-1}$, while GS242-01+11 has a diameter of $\sim 6^{\circ}$ and GS242-03+37 has a diameter of $16^{\circ}$ and spans the velocity range +33 to $+57 \mathrm{~km} \mathrm{~s}^{-1}$.

Stacey \& Jackson (1982, SJ from here onwards) surveyed the region delimited by $239^{\circ} \leq l \leq 251^{\circ}$ and $-9^{\circ} \leq b \leq+2^{\circ}$ every 0.2 in both $l$ and $b$. One of the most outstanding features at low velocities in this survey is the almost complete absence of HI in the velocity range -5 to $+10 \mathrm{~km} \mathrm{~s}^{-1}$ between the galactic longitudes $236^{\circ} \leq l \leq 250^{\circ}$ and galactic latitudes $-11^{\circ} \leq b \leq+1^{\circ}$. This huge region of low HI emissivity is known as the Puppis Window, because it has very low visual extinction (less than $2 \mathrm{mag}$ ) in the first $8 \mathrm{kpc}$ away from the Sun (Reed \& Fitzgerald 1984). This HI void and its surrounding ridge of HI emission has been reported by Heiles (1979) as the giant stationary HI shell GS241-04-05. SJ also report seven other HI shells. One of them, GS249+00+29, has an angular extent $(\triangle l \times \Delta b)$ of $4^{\circ} \times 5^{\circ}$ and is observed over the velocity range +24 to $+38 \mathrm{~km} \mathrm{~s}^{-1}$. Another giant shell listed by SJ, GS241-01+15, corresponds to the giant shell GS241-01+11 previously reported by Heiles (1979).

Dubner et al. (1992) find an extended HI circular structure 6.5 in radius, centred approximately at $(l, b)=\left(266^{\circ},-2.5\right)$, which shows a hole in the HI distribution at its centre, has a systemic velocity of $3 \pm 2 \mathrm{~km} \mathrm{~s}^{-1}$, and may be expanding at a velocity of 6 to $8 \mathrm{~km} \mathrm{~s}^{-1}$. This structure, referred to as the thick shell by Dubner et al. (1992), may be related to the Gum nebula. The same authors mention that the thick shell appears surrounded by a concentric $\mathrm{HI}$ feature about $13^{\circ}$ in radius. This feature, referred to as the disk by Dubner et al. (1992), has some correspondence in its northern edge with bright optical filaments.

A large disk, roughly $17^{\circ}$ in radius and centred at $(l$, $b)=\left(262^{\circ},-3^{\circ}\right)$ has been observed to agree in position and size with the optical image of the Gum nebula (Reynoso \& Dubner 1997). This disk, referred to as the giant HI disk by the authors, is best observed along the velocity interval +6 to $+10 \mathrm{~km} \mathrm{~s}^{-1}$. In the same paper, Reynoso \& Dubner (1997) draw attention to a giant shell, $14^{\circ}$ in radius, centred at $(l, b) \simeq\left(257^{\circ},-23^{\circ}\right)$, wich is visible along the velocity range -10 to $+14 \mathrm{~km} \mathrm{~s}^{-1}$. This feature reaches a maximum angular size at $+4 \mathrm{~km} \mathrm{~s}^{-1}$ and 
may have been created by the stellar winds of the O-type star HD 49798.

In connection with the Vela SNR, Dubner et al. (1998) find a thin and almost circular HI shell having a systemic velocity of $1.6 \pm 0.8 \mathrm{~km} \mathrm{~s}^{-1}$ and expanding at a velocity of $30 \pm 3 \mathrm{~km} \mathrm{~s}^{-1}$. This feature is centred at $(l, b)=\left(264^{\circ} .1,-1.6\right)$ and has an angular radius of $\sim 3.5$.

In a study aimed at detecting new HI shells located in the Southern Hemisphere, McClure-Griffiths et al. (2002) find a series of shells whose centres (as defined in their Table 1) cover the galactic coordinates range $255^{\circ} \leq l \leq 280$ and $-3^{\circ} \leq$ $b \leq 0^{\circ}$. The central radial velocities of these shells are higher than $+36 \mathrm{~km} \mathrm{~s}^{-1}$, have expansion velocities between 12 and $18 \mathrm{~km} \mathrm{~s}^{-1}$, and have an angular spatial extent of $2.2 \leq \Delta l \leq 5.4$ and $2.2 \leq \Delta b \leq 3.8$. Two of the newly-catalogued objects, namely GSH277+00+36 and GSH280+00+59 whose angular extent in $b$ is larger than $20^{\circ}$, are proposed as candidates for HI chimneys. All the giant shells reported by McClure-Griffiths et al. (2002) in the region of the IVS are located between 5.3 and $9.2 \mathrm{kpc}$ away from the Sun.

In summary quite a few HI structures are known to exist towards the region where the IVS is observed. The quoted distance for the IVS is about $450 \mathrm{pc}$ (Sahu 1992), therefore the signature of the neutral hydrogen that might be associated with it shoul be searched for at low radial velocities. Since some of the catalogued shells have central radial velocities that imply kinematical distances greater than $1.4 \mathrm{kpc}$, they will not be considered any further. On the other hand, the radial velocity of the thick shell, the disk, the giant $H_{I}$ disk, the HI associated with the Vela SNR, GS242-01+11, and GS241-04-05 are either slightly positive or slightly negative implying kinematical distances within $1 \mathrm{kpc}$ from the Sun.

Bearing in mind their location and angular coverage, it is clear that these structures will hinder the detection of any lowvelocity HI that may be related to the IVS, along its highlongitude $\left(l \geq 262^{\circ}\right)$ and high-latitude $\left(b \geq-9^{\circ}\right)$ extreme. Therefore, we restrict our efforts to uncovering a possible gaseous counterpart to the IVS by focusing our attention on those areas of the sky located outside the region covering by the features mentioned above (the area delimited by $l \leq 262^{\circ}$ and $b \leq-9^{\circ}$ ).

Within the area occupied by the IVS, our HI database shows that the Milky Way emission covers the velocity range from -30 to $+180 \mathrm{~km} \mathrm{~s}^{-1}$. Though the entire datacube, covering the region $245^{\circ} \leq l \leq 280^{\circ}$ and $-20^{\circ} \leq b \leq+5^{\circ}$, was inspected using the AIPS software package, some peculiarities in the HI gas distribution were found only at low velocities $\left(|v| \leq 30 \mathrm{~km} \mathrm{~s}^{-1}\right)$.

In Fig. 4 the mean Hi brightness temperature distribution covering the velocity range -31.9 to $+35 \mathrm{~km} \mathrm{~s}^{-1}$ is shown in six panels. Every image represents a mean of the HI emission over eleven individual images and covers a velocity range of $\sim 11.4 \mathrm{~km} \mathrm{~s}^{-1}$. In the velocity ranges from -31.9 to $-21.6 \mathrm{~km} \mathrm{~s}^{-1}$ (upper left panel) and -20.6 to $-10.3 \mathrm{~km} \mathrm{~s}^{-1}$ (upper right panel), most of the galactic HI emission is concentrated toward the high-longitude extreme of the images, as expected from standard galactic rotation models. Besides a low-level emission feature observed at $258^{\circ} .0 \leq l \leq 268^{\circ} .0$ and $b \sim-14^{\circ}$, in the -20 to $-10 \mathrm{~km} \mathrm{~s}^{-1}$ interval, there is very little morphological correspondence between the IVS (as delineated by the $100 \mu \mathrm{m}$ emission) and the HI distribution.

From -9.3 to $+1.0 \mathrm{~km} \mathrm{~s}^{-1}$ (middle left panel), although the bulk of the galactic emission is mostly seen at $l \geq 265^{\circ}$, two large regions of low HI emissivity are observed at $l \leq 265^{\circ}$. One of the HI voids, centred at $(l, b) \simeq\left(245^{\circ},-5^{\circ}\right)$, corresponds
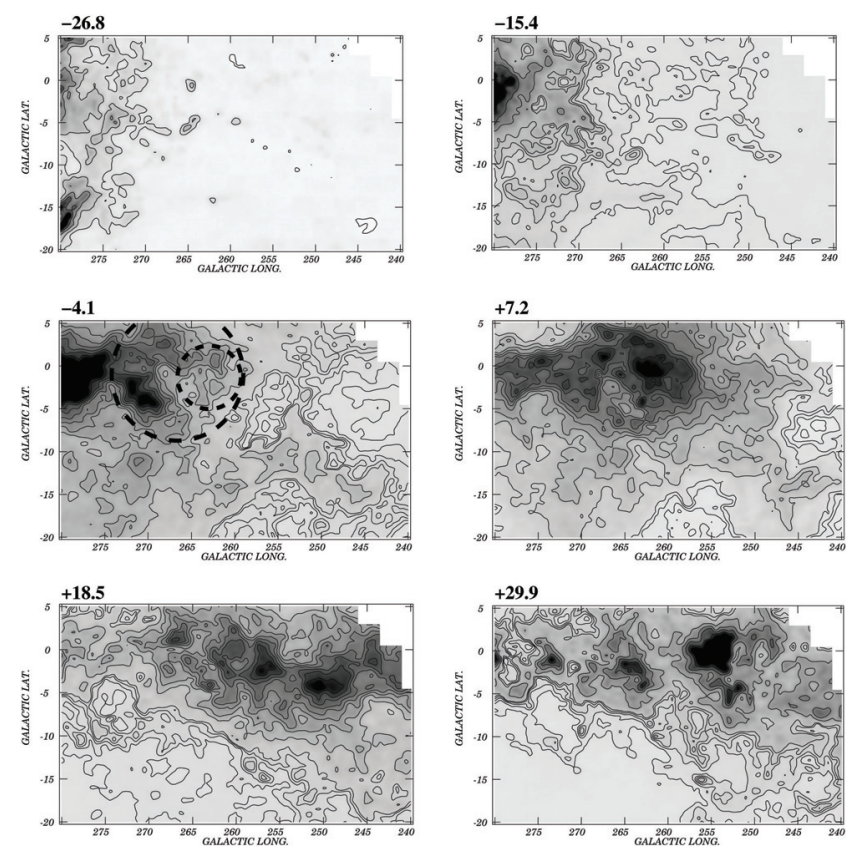

Fig. 4. Neutral hydrogen brightness-temperature images at different radial velocities. Each image is an average of eleven individual images. The central radial velocity of each image is indicated above the upper left corner. The lowest contour line is $0 \mathrm{~K}$ and the contour spacing is $2 \mathrm{~K}$ till $10 \mathrm{~K}$. From there onwards the contour spacing is $10 \mathrm{~K}$. In the first two images $\left(-26.8\right.$ and $\left.-15.4 \mathrm{~km} \mathrm{~s}^{-1}\right)$, the $1 \mathrm{~K}$ contour line is also drawn. The broken black circumferences seen in the image at $-4.1 \mathrm{~km} \mathrm{~s}^{-1}$ roughly indicate the area occupied by the HI emission related to the Vela SNR (small circumference, Dubner et al. 1998) and the thick shell (large circumference, Dubner et al. 1992).

to the Puppis Window. The other region of low emissivity is seen at $250^{\circ} \leq l \leq 262^{\circ}$ and $b \leq-13.5$, and it marks the location of the upper extreme of the HI void that may be related to the star HD 49798 (Reynoso \& Dubner 1997). The central HI hole of the thick shell (Dubner et al. 1992) is also visible at $(l, b)=\left(256^{\circ} .0,-2^{\circ} .5\right)$. Along $254^{\circ} \leq l \leq 271^{\circ}$ and $-14^{\circ} \leq b \leq-9^{\circ}$, an arc-shaped feature is observed. This feature appears as a collection of scattered HI peaks stretching from $(l, b) \simeq\left(271^{\circ} .0\right.$, $\left.-11^{\circ} .5\right)$ to $(l, b) \simeq\left(254^{\circ} .5,-9^{\circ} .5\right)$, and it reaches its southernmost position at $(l, b) \simeq\left(260^{\circ} .0,-14^{\circ} .0\right)$. From +2.1 to $+12.4 \mathrm{~km} \mathrm{~s}^{-1}$ (middle right panel), the main features described above remain visible, except for the arc-shaped feature that is now barely observable. Finally, along the velocity range +13.4 to $+35.0 \mathrm{~km} \mathrm{~s}^{-1}$ (lower left panel and lower right panel, respectively), the bulk of the HI emission shifts towards negative latitudes following the warp of the galactic plane.

Based on the relative angular location of the different HI features with respect to the location of the IVS (see Fig. 4), it is likely that the atomic gas probably associated with the IVS may be present in the velocity range -20.6 to $+1.0 \mathrm{~km} \mathrm{~s}^{-1}$. In order to follow its spatial distribution in more detail, a montage of ten images is shown in Fig. 5. They cover the velocity range from -23.7 to $+4.1 \mathrm{~km} \mathrm{~s}^{-1}$, and every image represents a mean of the emission over a velocity interval of $\sim 3.1 \mathrm{~km} \mathrm{~s}^{-1}$.

At $-23.7 \mathrm{~km} \mathrm{~s}^{-1}$ the bulk of the emission is concentrated at $l \geq 270^{\circ}$ and shows the existence of several holes and HI maxima of varying sizes. The concentration of the HI emission on the high-longitude extreme of the image is the one expected based 

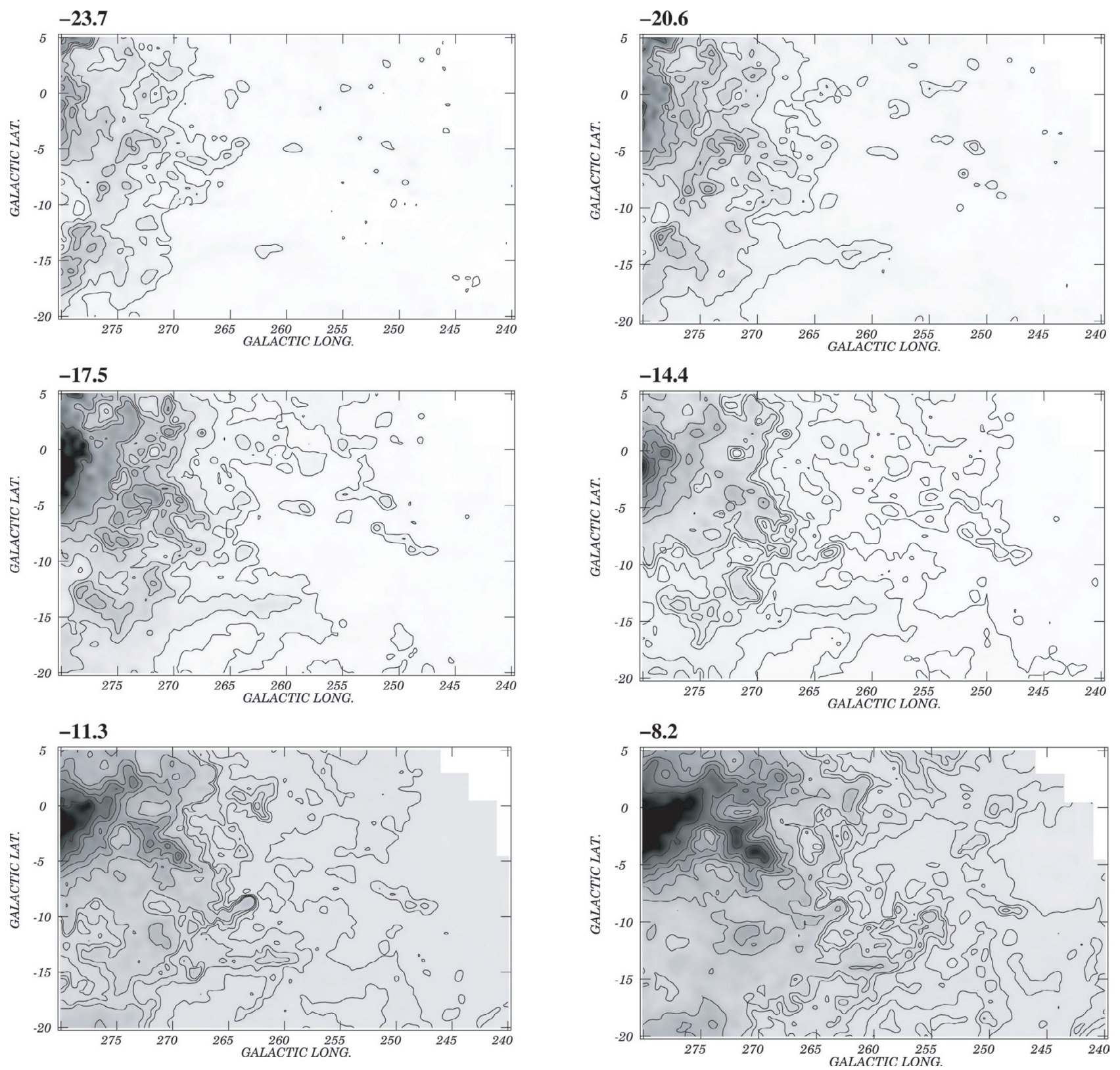

Fig. 5. Mosaic of HI mean brightness-temperature images for the velocity interval -23.7 to $+4.1 \mathrm{~km} \mathrm{~s}^{-1}$. Each image is an average of three individual images. The central velocity of each image is indicated in the upper left corner. The lowest contour line is $0 \mathrm{~K}$ and the contour spacing is $2 \mathrm{~K}$ to $10 \mathrm{~K}$. From there onwards the contour spacing is $10 \mathrm{~K}$. In those images at $-23.7,-20.6,-17.5$, and $-14.4 \mathrm{~km} \mathrm{~s}^{-1}$, the $1 \mathrm{~K}$ contour line is also drawn.

on standard galactic rotation models. On every image of Fig. 5, the northern border of the IAR HI survey can be seen in the upper right hand corner.

From -20.6 to $-14.4 \mathrm{~km} \mathrm{~s}^{-1}$, low-intensity Hi emission with irregular spatial distribution covers an increasing area of the lowlongitude extreme of the images as the radial velocity increases. A bar-like feature that is observed between $259^{\circ} \leq l \leq 268^{\circ}$ and $b \simeq-14^{\circ}$ gets brighter and broader as we move towards positive velocities. This feature appears projected onto a region of the sky that is close to the IVS.

At $-11.3 \mathrm{~km} \mathrm{~s}^{-1}$, the overall $\mathrm{HI}$ emission depicts the same general trend observed at lower velocities, namely strong at $l \geq 270^{\circ}$ and weak and scattered at $l \leq 270^{\circ}$. Besides the bar-like ridge of $\mathrm{HI}$ emission mentioned above, two regions of low emissivity set in, namely the low-velocity end of GS241-04-05, seen at $240^{\circ} \leq l \leq 248^{\circ}$ and $-9^{\circ} \leq b \leq+2^{\circ}$, and the upper part of the
HI, avoid created by HD 49798, which begins to be detectable at $(l, b) \simeq\left(252^{\circ},-19^{\circ}\right)$.

From -8.2 to $+4.1 \mathrm{~km} \mathrm{~s}^{-1}$, the HI deficiencies related to both HI holes become more noticeable and reach their maximum angular size in the range $-2 \leq v \leq+1 \mathrm{~km} \mathrm{~s}^{-1}$. A weak arcshaped feature is observed at -8.2 and $-5.2 \mathrm{~km} \mathrm{~s}^{-1}$. This feature gets stronger as we move towards positive velocities, and in the $-5.2 \mathrm{~km} \mathrm{~s}^{-1}$ image, it can be traced from $(l, b) \simeq\left(271^{\circ}\right.$, $\left.-11^{\circ}\right)$ to $(l, b) \simeq\left(254^{\circ},-9^{\circ}\right)$. This feature may be the counterpart at higher positive radial velocities of the bar-like feature seen at higher negative velocities, or it could be part of an HI shell that may be surrounding either GS241-04-5 or the void around HD 49798. At $-5.2 \mathrm{~km} \mathrm{~s}^{-1}$ a ridge of HI emission starts to develop between $(l, b) \simeq\left(253^{\circ},-12^{\circ}\right)$ and $(l, b) \simeq\left(245^{\circ},-17^{\circ}\right)$. As we move towards higher positive velocities, this ridge achieves a higher mean brightness temperature and becomes thicker. 

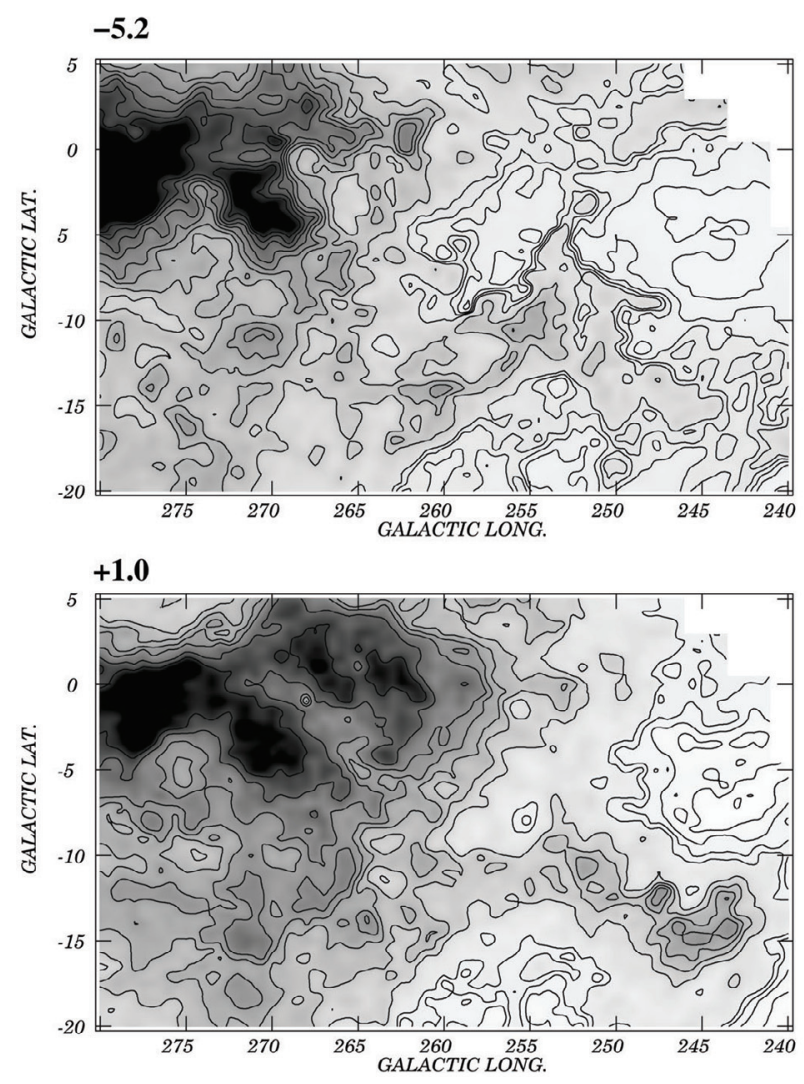

Fig. 5. continued.

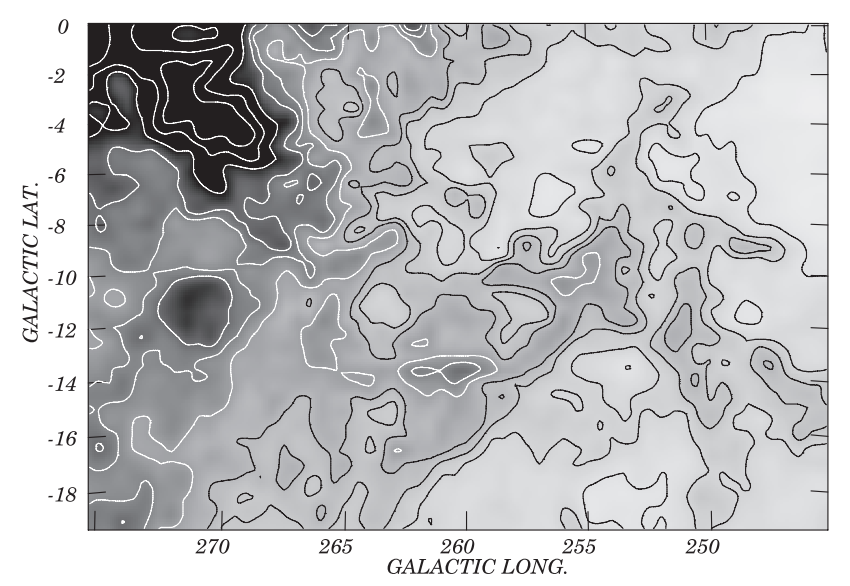

Fig. 6. Neutral hydrogen brightness-temperature average map along the velocity range -17.5 to $-2.06 \mathrm{~km} \mathrm{~s}^{-1}$. The lowest contour is $2 \mathrm{~K}$ and the contour spacing is $2 \mathrm{~K}$ to $8 \mathrm{~K}$. Above that contour line, the step is $4 \mathrm{~K}$ to $20 \mathrm{~K}$, and from there onwards the contour step is $10 \mathrm{~K}$. The gray scale spans the range from -2 (white) to 30 (black) K.

At -2.1 and $+1.0 \mathrm{~km} \mathrm{~s}^{-1}$, both GS241-04-05 and the void around HD 49798 are completely surrounded by thick walls of HI emission. In these images, it is very hard to disentangle the HI emission that may arise from the arc-shaped feature observed at lower velocities from the one that may be arising from HI shells surrounding GS241-04-05 and/or the void around HD 49798. The HI shells surrounding these objects have a lower mean brightness temperature and tend to merge with the overall galactic emission.

In order to enhance those Hi features that may be related to the IVS, a smooth HI background was removed from the original
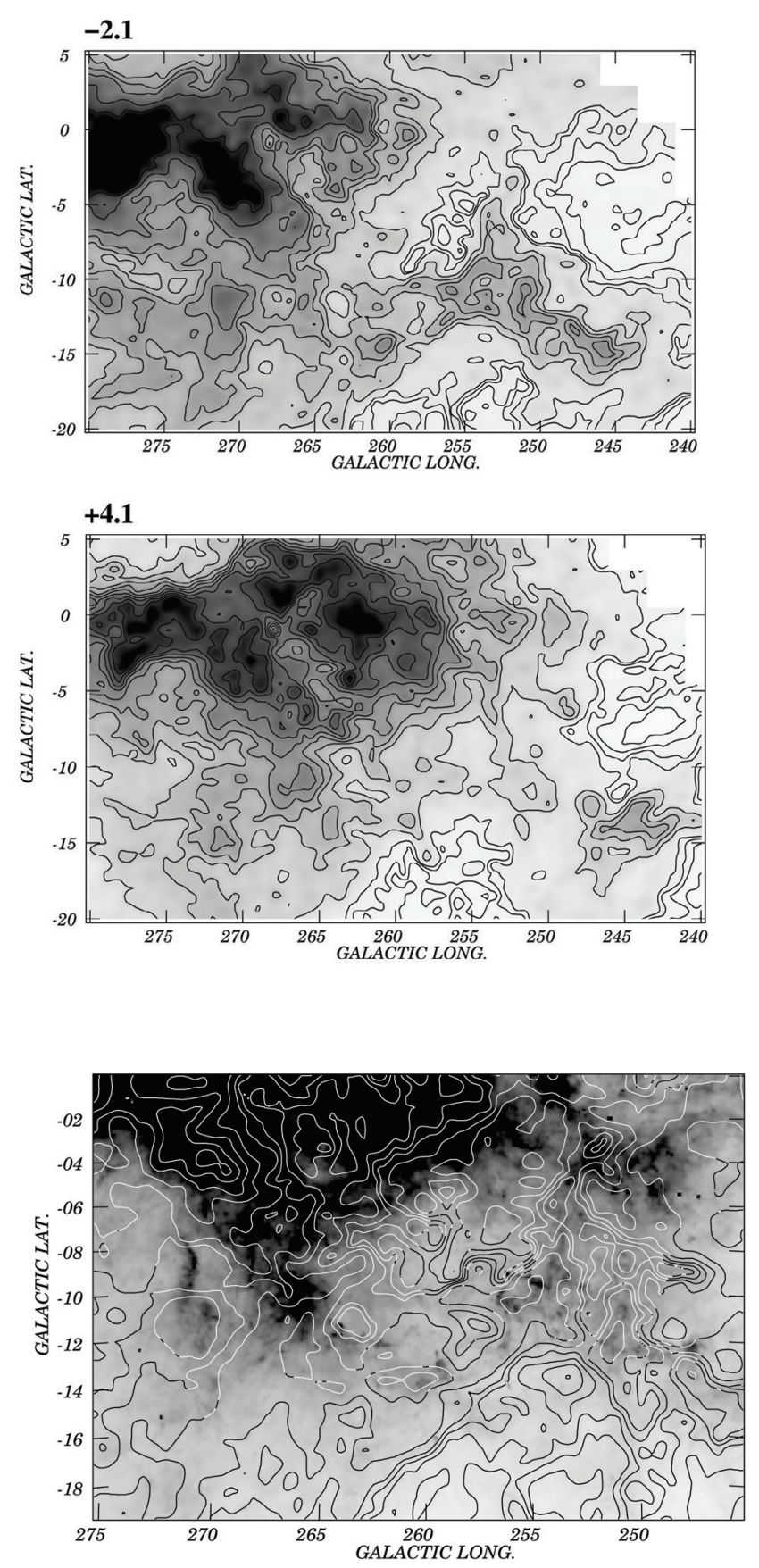

Fig. 7. Neutral hydrogen brightness-temperature image (as in Fig. 6) overlaid on a $100 \mu \mathrm{m}$ IRAS image (grey scale).

HI images. To this end, every individual HI line image along the velocity interval -15 to $+6 \mathrm{~km} \mathrm{~s}^{-1}$ was spatially convolved with a Gaussian beam having a fullwidth half-maximum $(\equiv \phi)$ of $4^{\circ}$. This new image was substracted from the observed one at the same velocity. The resulting image, known as a residual image, mainly contains structures whose angular sizes cover the range between 0.5 (the angular resolution of the HI survey) and $\phi$. This procedure, similar to the one used by Sofue \& Reich (1979) for processing continuum data, was repeated until the difference between two consecutive residual images was below a certain limit. In Fig. 6 an average of twelve spatially-filtered consecutive images, covering the velocity range from -17.5 to $-2.06 \mathrm{~km} \mathrm{~s}^{-1}$ is shown. In Fig. 7 the same HI distribution is overlaid on the $100 \mu \mathrm{m}$ IVS image (grey scale). There is a remarkable spatial 


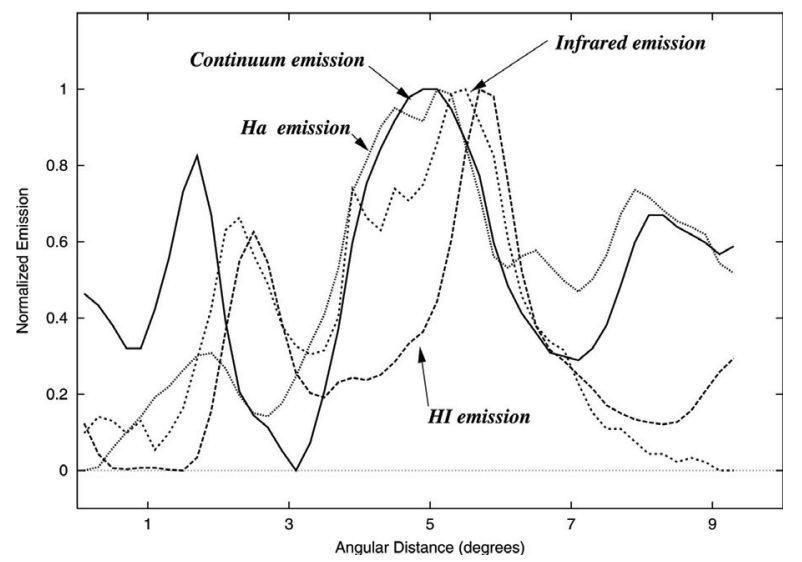

Fig. 8. Radial plots of the mean emission at $1420 \mathrm{MHz}$ (continuum), $100 \mu \mathrm{m}$ (infrared), $\mathrm{H}_{\alpha}$, and $\mathrm{HI}$ line frequencies. Each data point represents the normalized mean emission value on a ring $12^{\prime}$ wide between the position angles $162^{\circ}$ and $265^{\circ}$. The peak emissions used to normalize this cuts are $97 \mathrm{mK}$ at 1420 continuum, $9.3 \mathrm{MJy} / \mathrm{sr}$ at $100 \mu \mathrm{m}$, $56.9 \mathrm{~K}$ for the $\mathrm{HI}$ line, and $795 \mathrm{R}$ for $\mathrm{H}_{\alpha}$. The abscissae are mean angular distances expressed in degrees of the different $12^{\prime}$ rings from the point $(l, b)=(259.85,-8.25)$

correlation among the features delineating the IVS at $100 \mu \mathrm{m}$ (grey scale) and several of the small scale HI structures (solid contours).

Under the assumption of an optically thin HI emission, the HI mass of the neutral hydrogen feature shown in Fig. 6, is of the order of $\sim 1.2 \times 10^{4} d^{2} M_{\odot}$, where $d$ is the distance expressed in kpc. Since the area where the atomic hydrogen was identified corresponds to about $\sim 25 \%$ of the total extent of the IVS, assuming that there is HI line emission at a similar column density over all the unexplored part of the IVS, then the total atomic mass of gas would be $\sim 6.8 \times 10^{4} d^{2} M_{\odot}$. To derive this figure, a relative helium abundance of $34 \%$ by mass was assumed.

\section{Discussion}

\subsection{A gaseous counterpart to the IVS}

Figures 2, 3, and 7 show that there is a reasonable spatial correlation between ionized and atomic gas with the IR emission arising from heated dust grains.

To further illustrate the spatial correlation among the different emissions analysed in this paper, normalized radial distribution of the emission arising from heated dust $(100 \mu \mathrm{m})$, ionized gas (1420 continuum), optical emission $\left(\mathrm{H}_{\alpha}\right)$, and atomic gas (HI line) are shown in Fig. 8. The origin of the angular distance was set to $(l, b)=\left(259^{\circ} .85,-8^{\circ} .25\right)$, which is the centroid of the IVS as determined from the observed IR emission. Every point in the cross-cuts shown in Fig. 8 represents a mean value of the corresponding emission along concentric rings spaced by $12^{\prime}$. In order to avoid most of the confusing effects that may arise from unrelated material, the numerical integration of the different emissions was only performed between position angles $\sim 162^{\circ}$ and $\sim 265^{\circ}$ (measured from north counterclockwise). To obtain the cross-cuts shown in Fig. 8, at each wavelength, the corresponding minimum value of emission was substracted from the observed cross-cut values and later on normalized to the maximum remanining value. The maximum and minimum emission along the observed cross-cuts are 167.8 and $70.8 \mathrm{mK}$ for the $1420 \mathrm{MHz}$ continuum, 21.6 and 12.3 MJy/sr at $100 \mu \mathrm{m}, 56.3$ and $-0.6 \mathrm{~K}$ for the HI line, and 1253.0 and 458.6 Rayleighs ${ }^{4}$. The mean weighted (by intensity) emission radius is $4.76 \pm 0.16$ for the $\mathrm{H} \alpha$ emission, $4^{\circ} 90 \pm 0.18$ for the continuum, $5^{\circ} .05 \pm 0.20$ for the $100 \mu \mathrm{m}$ emission, and $5^{\circ} .72 \pm 0.26$ for the neutral hydrogen. This trend agrees with the one expected for a shell-like mass distribution that is being ionized from inside. The full-width half maximum of the shell is, within errors, quite similar for the continuum, $100 \mu \mathrm{m}$, and $\mathrm{H}_{\alpha}$ emission, namely $\sim 2.4$, but is considerably narrower for the $\mathrm{HI}\left(\sim 1^{\circ} .2\right)$.

The HI emission likely to be related to the IVS covers at least the velocity range from -17.5 to $-2.1 \mathrm{~km} \mathrm{~s}^{-1}$. Molecular gas belonging to this feature may have been detected in the $1667 \mathrm{MHz} \mathrm{OH}$ line (Woermann et al. 2001). Those four $\mathrm{OH}$ spectra that fall right on top of the HI arcshaped feature show $\mathrm{OH}$ molecular-line emission in the velocity range $-2.2 \leq v \leq-1.2 \mathrm{~km} \mathrm{~s}^{-1}$. Slightly negative radial velocities are also observed towards the western edge of the IVS at $(l, b)=\left(255^{\circ} .9,-9.2\right)$ in a region that contains the CG 8, 9, and 10. This region has been studied by different authors (Gredel et al. 1994; Churchwell et al. 1996), who found molecularline emission covering a narrow range around $-4 \mathrm{~km} \mathrm{~s}^{-1}$. In their study of the distribution and kinematics of the molecular clouds in the Gum nebula, Yamaguchi et al. (1999) found (see their Fig. 9) molecular concentrations that closely follow the IVS. Furthermore, the radial velocity of these concentrations are mostly located in the velocity interval -10 to $+10 \mathrm{~km} \mathrm{~s}^{-1}$ (see their Fig. 2, panels a) and b)). Thus, the atomic gas (HI) and the molecular gas emission at a specific number of positions along the HI arc-shaped feature, agree with each other.

In view of the good spatial correlation between the ionized gas and the $100 \mu \mathrm{m}$ IRAS emission (Fig. 2), between the $\mathrm{H}_{\alpha}$ and radio continuum emission (Fig. 3), and between HI and $100 \mu \mathrm{m}$ emission (Fig. 7), it would be difficult to ascribe these morphological agreements to projection effects of otherwise spatially unrelated objects. Thus, we believe these four different emissions, namely $\mathrm{H}_{\alpha}, 100 \mu \mathrm{m}, 1.4 \mathrm{GHz}$ continuum emission, and the HI line, are showing the spatial distribution of different constituents of the same object.

\subsection{Energetics}

In connection with the stellar objects known to be present in this region of the sky, two outstanding stars, namely $\gamma^{2}$ Vel $\left(l=262^{\circ} .8, b=-7.7\right)$ and $\zeta$ Pup $\left(l=255^{\circ} .98, b=-4.71\right)$, lie within (or close to) the borders of the IVS. The former is a Wolf-Rayet (WC 8) spectroscopic binary, while $\zeta$ Pup is a likely run-away O4If star. The spectral type of the secondary star of $\gamma^{2}$ Vel is a matter of some debate. It was originally classified as an O9 supergiant (Conti \& Smith 1972), though recent works point towards an earlier spectral type and a different luminosity class (O7.5 III-V, ESA 1997; O8 III, van der Hucht et al. 1997).

The parallax of $\gamma^{2} \mathrm{Vel}$, as measured by Hipparcos, corresponds to a distance of $258_{-31}^{+41}$ pc (van der Hucht et al. 1997). Pozzo et al. (2000) have cast some doubts on this determination, and locate $\gamma^{2} \mathrm{Vel}$ at a distance of $410 \pm 10 \mathrm{pc}$. The distance to $\zeta$ Pup is $429_{-77}^{+120} \mathrm{pc}$, based on the parallax measured by Hipparcos (van der Hucht et al. 1997). Besides these stars, two conspicuous stellar aggregates are also located in the area, namely the OB association Vel OB2 and the open cluster $\operatorname{Tr} 10$. Based on the mean parallax of 93 members of Vel OB2, its distance turns out to be $\sim 410 \pm 40$ pc (de Zeeuw et al. 1999). The members of the association are concentrated on the sky around $(l, b)=\left(263^{\circ},-7^{\circ}\right)$

\footnotetext{
${ }^{4} 1$ Rayleigh $=10^{6} / 4 \times \pi$ photons $/ \mathrm{cm}^{2} / \mathrm{s} / \mathrm{sr}$.
} 
within a radius of $5^{\circ}$, and most of them lie within the IVS (see Fig. 11 of de Zeeuw et al. 1999). The earliest spectral type on the main sequence is B1, suggesting an upper limit for the age of $\sim 10^{7}$ yr. Sahu \& Blaauw (1993) mention that this association is $\sim(2-3) \times 10^{7} \mathrm{yr}$ and on the verge of disintegration. The other stellar aggregate, $\operatorname{Tr} 10$, has a total of 23 catalogued stars, the earliest one being of spectral type B3 V. It has an age of $\sim 1.5 \times 10^{7} \mathrm{yr}$ and is located at a distance of $366 \pm 23 \mathrm{pc}$ (de Zeeuw et al. 1999). The central position of the cluster is $(l, b)=\left(262^{\circ} .8,0.64\right)$, and its members are spread over $\sim 8^{\circ}$ in the sky.

If the IVS were expanding, as claimed by Sahu \& Sahu (1993) and Rajagopal \& Srinivasan (1998), the ultimate cause of the expanding motions could be the injection into the ISM of mechanical energy via stellar winds and/or supernova explosions by the early type stars known to exist along this galactic line of sight. Based on their distances and angular locations with respect to the centroid of the IVS, the OB association Vel OB2, the open cluster $\operatorname{Tr} 10$, and the stars $\gamma^{2} \mathrm{Vel}$ and $\zeta$ Pup are the most promising sources of energy to account for the expanding motions.

In order to test the hypothesis that at least one of the abovementioned objects may be the physical cause of the expansion of the IVS, we have to evaluate both the kinetic energy of the expanding gas and the mechanical luminosity of the wind, $L_{\mathrm{w}}=0.5 \times \dot{M} \times v_{\infty}^{2}$, injected into their surrounding ISM by the different stellar objects.

In obtaining the total IVS mass, we have to add up the ionized, atomic, and molecular mass. In this respect, based on the 60- and $100 \mu \mathrm{m}$-integrated fluxes, and assuming a gas-to-dust ratio of 100, Sahu (1992) estimated that the total mass of gas, mostly in molecular form, would be around $\sim 10^{6} M_{\odot}$. On the other hand, based on $2.6 \mathrm{~mm}$ CO line observations towards a sample of IRAS point sources likely to be embedded in the IVS, and assuming an IVS distance of $\sim 500 \mathrm{pc}$, Rajagopal \& Srinivasan (1998) claim that the amount of molecular gas associated with it is $\sim 10^{5} M_{\odot}$.

Adopting the mass of molecular gas determined by Rajagopal \& Srinivasan (1998) and taking the ionized and atomic components derived from our observations into account, the total amount of gas is $\sim 10^{5} M_{\odot}$. A distance of $400 \mathrm{pc}$ was assumed in these calculations. Thus, the total expansion energy of the IVS, assuming an expansion velocity of $13 \mathrm{~km} \mathrm{~s}^{-1}$, is $1.7 \times 10^{50} \mathrm{erg}$.

We attempt now to derive the mechanical luminosity injected into the ISM by the stellar objects $\gamma^{2}$ Vel, $\zeta$ Pup, Vel OB2, and $\operatorname{Tr} 10$. The mass-loss rate and wind terminal velocity of the WC8 star of $\gamma^{2}$ Vel are $\dot{M} \sim 2.8 \times 10^{-5} M_{\odot} \mathrm{yr}^{-1}$ and $v_{\infty} \sim 1450 \mathrm{~km} \mathrm{~s}^{-1}$, respectively (Schaerer et al. 1997). The mechanical luminosity of the wind is $L_{\mathrm{w}} \sim 1.9 \times 10^{37} \mathrm{erg} \mathrm{s}^{-1}$. Assuming a typical duration of the WR phase of $\sim 5 \times 10^{5} \mathrm{yr}$ (Maeder 1991), the injected kinetic energy of the WR star amounts to $3 \times 10^{50} \mathrm{erg}$. Since $\gamma^{2}$ Vel is a binary system, to obtain a rough estimate of the total mechanical luminosity injected into the ISM by this system, we must also consider the contribution of the presentday members of the binary system, along their previous evolutive stages. Bearing in mind the spectral classification of the secondary star and following Massey (1998), we may guess that the secondary star very likely had a main-sequence spectral type falling in the range O5-O8, whilst the main-sequence spectral type of the present WR star had to be earlier. For the purpose of our calculations, we adopt the same spectral type for both stars (either $05 \mathrm{~V}$ or $\mathrm{O} 8 \mathrm{~V}$ ). Adopting the mechanical luminosities given by Leitherer (1998) and assuming main-sequence lifetimes of $4 \times 10^{6} \mathrm{yr}(\mathrm{O} 5 \mathrm{~V})$ or $6.4 \times 10^{6} \mathrm{yr}(\mathrm{O} 8 \mathrm{~V})$, the overall contribution of the $\gamma^{2}$ Vel system is about $(5-11) \times 10^{50} \mathrm{erg}$.

Next, we consider the possible contribution from Vel OB2. In doing so, we only take those members into account whose spectral type on the main sequence is earlier than B3V. We adopted this criterion based on the fact that the mechanical energy injected by a late B-type star is negligible compared to that of a star having an earlier spectral type (e.g. $\left.L_{\mathrm{w}}(\mathrm{B} 2 \mathrm{~V}) \simeq 15 \times L_{\mathrm{w}}(\mathrm{B} 5 \mathrm{~V})\right)($ Leitherer 1998). In computing the individual contribution to the total mechanical energy, mass-loss rates were derived following Lamers \& Leitherer (1993), while terminal velocities were derived from Leitherer et al. (1992). Thereupon, the present day rate of mechanical energy input into the ISM by VelOB2 is $\sim 10^{35} \mathrm{erg} \mathrm{s}^{-1}$. This would amount to $\sim(6-9) \times 10^{49}$ erg depending on the assumed lifetime of the OB association. $\operatorname{Tr} 10$ would contribute with a mechanical luminosity of $\sim 3 \times 10^{33} \mathrm{erg} \mathrm{s}^{-1}$. This is equivalent to a total mechanical input of $1.4 \times 10^{48} \mathrm{erg}$. If the theoretical kinetic energyefficiency conversion of up to $20 \%$ (for an energy conserving insterstellar bubble) were adopted, only $\gamma^{2}$ Vel would be powerful enough to explain the IVS expansion via stellar winds . On the other hand, if the observed efficiency conversion $(\sim 3 \%)$ were used in our calculations, not even the joint action of $\gamma^{2}$ Vel and Vel OB2 could account for the expanding motions. In this case some extra injection of energy (e.g. a supernova explosion) is needed. It is worth mentioning that Hoogerwerf et al. (2001) claims that HD 64760, a B0.5Ib run away star, was located inside the Vel OB2 association some $6 \mathrm{Myr}$ ago. They suggest a binary-supernova scenario to explain its origin.

\section{Summary}

Mostly using data collected by the $30 \mathrm{~m}$ dishes of the Instituto Argentino de Radioastronomía, we carried out a study of the spatial distribution of the neutral hydrogen and radio continuum emission at $1420 \mathrm{MHz}$ in the region of the IVS. These data revealed for the first time the existence of arc-shaped features having a good morphological correlation with the IVS (as traced by its $100 \mu \mathrm{m}$ emission). The centroid of both emissions is located at $(l, b)=\left(259^{\circ} .9,-8^{\circ} .3\right)$, wich is different from the one quoted by Sahu (1992), namely $(l, b)=\left(263^{\circ},-7^{\circ}\right)$.

The neutral hydrogen associated with the IVS is observed along the velocity interval -17.5 to $-2.1 \mathrm{~km} \mathrm{~s}^{-1}$. These velocities are in good agreement with radial velocities derived from molecular line observations carried out at specific locations along the HI feature we claim is related to the IVS. The radial distribution of the different emissions is consistent with a picture of a shell-like spatial distribution that is being ionized from inside. The radio continuum emission is mostly thermal in nature and is distributed as an arc-shaped feature having a radius of $\sim 4.9$ and a full-width half maximum of $\sim 2^{\circ} .4$. The neutral gas, as traced by the $\mathrm{HI}$ emission, is located further out (at a radius of $\sim 5^{\circ} .7$ ) and has a much narrower angular distribution; it is only 1.2 broad. Assuming a distance of $400 \mathrm{pc}$, the amount of ionized and atomic gas associated with the IVS are $2.3 \times 10^{4}$ and $6.8 \times 10^{4}$ solar masses, respectively.

The ultimate cause of the expansion of the IVS could not be identified, for it depends critically upon the efficiency of the conversion of wind mechanical luminosity into kinetic energy of the swept-up interstellar medium. If the canonical theoretical value of $20 \%$ is applied, then $\gamma^{2} \mathrm{Vel}$ is strong enough to give rise to the observed expanding motions. On the other hand, if the efficiency is close to the observed value $(\leq 3 \%)$, the combined action of the stellar winds of $\gamma^{2}$ Vel and Vel OB2 cannot account 
for the IVS expansion, so some extra source of energy injection (e.g. a supernova explosion) is needed.

Acknowledgements. This work was partially supported by the Argentinean National Research Council (CONICET) under projects PIP 4253/96 and PIP $2277 / 00$. The authors wish to thank the referee, Dr. B. Burton, for his comments and suggestions that greatly improved the paper.

\section{References}

Arnal, E. M., Bajaja, E., Larrarte, J. J., Morras, R., \& Pöppel, W. G. L. 2000, A\&AS, 142,35

Bajaja, E., Arnal, E. M., Larrarte, J. J., et al. 2005, A\&A, 440, 767

Chaisson, E. J. 1976, in Frontiers of Astrophysics, ed. E. H. Avrett (Cambridge Univ. Press), 259

Churchwell, E., Winnberg, A., Cardelli, J., Cooper, G., \& Suntzeff, N. B. 1996, ApJ, 469, 209

Cichowolski, S., Pineault, S., Arnal, E. M., et al. 2001, AJ, 122, 1938

Conti, P. S., \& Smith, L. F. 1972, ApJ, 172, 623

de Zeeuw, P. T., Hoogerwerf, R., de Bruijne, J. H. J., Brown, A. G. A., \& Blaauw, A. 1999, AJ, 117, 354

Dubner, G., Giacani, E., Cappa de Nicolau, C., \& Reynoso, E. 1992, A\&AS, 96, 505

Dubner, G. M., Green, A. J., Goss, W. M., Bock, D. C. J., \& Giacani, E. 1998, AJ, 116, 813

Duncan, A. R., Stewart, R. T., Haynes, R. F., \& Jones, K. L. 1995, MNRAS, 277, 36

ESA 1997, The Hipparcos and Tycho Catalogues, The Hipparcos Catalogue, ESA SP-1200

Feitzinger, J. V., \& Stüwe, J. A. 1984, A\&AS, 58, 365

Gaustad, J. E., McCullough, P. R., Rosing, W., \& Van Buren, D. 2001, PASP, 113,1326

Gredel, R., van Dishoeck, E. F., \& Black, J. H. 1994, A\&A, 285, 300

Haslam, C. G. T., Stoffel, H., Salter, C. J., \& Wilson, W. E. 1982, A\&AS, 47, 1

Heiles, C. 1979, ApJ, 229, 533

Hippelein, H. H., \& Weinberger, R. 1975, A\&A, 43, 405

Hoogerwerf, R., de Bruijne, J. H. J., \& de Zeeuw, P. T. 2001, A\&A, 365, 49

Kalberla, P. M. W., Burton, W. B., Hartmann, D., et al. 2005, A\&A, 440, 775
Lamers, H. J. G. L. M., \& Leitherer, C. 1993, ApJ, 412, 771

Leitherer, C. 1998 in Stellar Astrophysics for the Local Group, VIII Canary Islands Winter School of Astrophysics, ed. A. Aparicio, A. Herrero, \& F. Sánchez (Cambridge; New York: Cambridge University Press), 527

Leitherer, C., Robert, C., \& Drissen, L. 1992, ApJ, 401, 596

McClure-Griffthis, N. M., Dickey, J. M., Gaensler, B. M., \& Green, A. J. 2002, ApJ, 578, 176

Maeder, A. 1991 in WR stars and interrelations with other massive stars in galaxies, ed. K. A. van der Hucht, \& B. Hidayat, IAU Symp., 143, 445

Massey, P. 1998, in The Stellar Initial Mass Function, 38th Herstmonceux Conference, ed. G. Gilmore, \& D. Howell, ASP Conf. Ser., 142, 17

Mezger, P. G., \& Henderson, A. P. 1967, ApJ, 147, 171 (MH)

Murphy, D. C., \& May, J. 1991, A\&A, 247, 202

Pozzo, M., Jeffries, R. D., Naylor, T., et al. 2000, MNRAS, 313, L23

Prusti, T. 1992, Ph.D. Thesis, University of Groningen, The Netherlands

Rajagopal, J., \& Srinivasan, G. 1998, JApA, 19, 79

Reed, B. C., \& Fitzgerald, M. P. 1984, MNRAS, 211, 235

Reich, P., Testori, J. C., \& Reich, W. 2001, A\&A, 376, 861

Reipurth, B. 1983, A\&A, 117, 183

Reynolds, R. J. 1976a, ApJ, 203, 151

Reynolds, R. J. 1976b, ApJ, 206, 679

Reynoso, E. M., \& Dubner, G. M. 1997, A\&AS, 123, 31

Sahu, M. S. 1992, Ph.D. Thesis, University of Groningen

Sahu, M. S., \& Blaauw, A. 1993, in Massive Stars: Their Lives in the Interstellar Medium, ed. J. P. Cassinelli, \& E. B. Churchwell, ASP Conf. Ser., 35, 278

Sahu, M. S., \& Sahu, K. C. 1993, A\&A, 280, 231

Schaerer, D., Schmutz, W., \& Grenon, M. 1997, ApJ, 484, L153

Sofue, Y., \& Reich, W. 1979, A\&AS, 38, 251

Stacey, J. G., \& Jackson, D. D. 1982, A\&AS, 50, 377 (SJ)

Testori, J. C. 2001, Ph.D. Thesis, La Plata University

Testori, J. C., Reich, P., Bava, J. A., et al. 2001, A\&A, 368, 1123

van der Hucht, K. A., Schrijver, H., Stenholm, B., et al. 1997, New Astron., 2, 245

Wallerstein, G., Jenkins, E. B., \& Silk, J. 1980, ApJ, 240, 834

Weaver, H., \& Williams, D. R. W. 1973, A\&AS, 8, 1

Woermann, B., Gaylard, M. J., \& Otrupcek, R. 2000, MNRAS, 315, 241

Woermann, B., Gaylard, M. J., \& Otrupcek, R. 2001, MNRAS, 325, 1213

Yamaguchi, N., Mizuno, N., Moriguchi, Y., et al. 1999, PASJ, 51, 765

Zealey, W. J. 1979, New Zealand J. Sci., 22, 549 